\title{
miR-379-5p inhibits cell proliferation and promotes cell apoptosis in non-small cell lung cancer by targeting $\beta$-arrestin-1
}

\author{
YONGHONG JIANG $^{1 *}$, PANPAN ZHU ${ }^{1 *}$, YAMEI GAO ${ }^{2}$ and AIPING WANG ${ }^{1}$ \\ Departments of ${ }^{1}$ Second Inpatient Area of Oncology Surgery and ${ }^{2}$ Nursing, \\ Weinan Central Hospital, Weinan, Shaanxi 714000, P.R. China
}

Received November 11, 2019; Accepted April 28, 2020

DOI: $10.3892 / \mathrm{mmr} .2020 .11553$

\begin{abstract}
Lung cancer is the most common fatal type of cancer, demonstrating high incidence rates in both sexes. Therefore, it is of vital importance to devise more effective and targeted therapies to improve the treatment quality for patients. The present study aimed to determine the effects of microRNA (miR)-379-5p on cell proliferation and apoptosis, in addition to its underlying molecular mechanisms in lung cancer. Tumor and adjacent normal tissues were obtained from patients with NSCLC and transfection experiments in A549 cells were performed using miR-379-5p mimics and pcDNA3.1- $\beta$-arrestin-1 (ARRB1) overexpression plasmids . The cell proliferation rate was determined using a Cell Counting Kit- 8 assay and the cell apoptotic rate was analyzed using flow cytometry. Additionally, the mRNA and protein expression levels of proliferation-related signaling (PI3K, p-PI3K, AKT and p-AKT) and apoptotic-related factors (Bcl-2, Bax and caspase-3) were detected using reverse transcription-quantitative PCR and western blotting, respectively. The results of the present study revealed that miR-379-5p expression levels were downregulated, whereas ARRB1 expression levels were significantly upregulated in NSCLC tissues and cell lines. Following the successful transfection of the miR-379-5p mimic and ARRB1 overexpression plasmid, it was revealed that the overexpression of miR-379-5p inhibited cell proliferation and promoted cell apoptosis, whereas ARRB1 overexpression reversed this inhibition over proliferation and promotion of apoptosis. The increased cell apoptotic rate observed in the miR-379-5p mimics group was associated with a significant downregulation and upregulation of Bcl-2, and Bax and caspase- 3 expression levels, respectively. Finally,
\end{abstract}

Correspondence to: Dr Aiping Wang, Department of Second Inpatient Area of Oncology Surgery, Weinan Central Hospital, 7 Shengli Street, Weinan, Shaanxi 714000, P.R. China

E-mail: aiping.wang@yandex.com

${ }^{*}$ Contributed equally

Key words: non-small cell lung cancer, proliferation, apoptosis, microRNA-379-5p, $\beta$-arrestin-1
ARRB1 was identified as a target gene of miR-379-5p. In conclusion, the expression levels of miR-379-5p were demonstrated to be significantly downregulated in lung cancer. In addition, miR-379-5p overexpression led to the decreased expression levels of Bcl-2, phosphorylated (p)-PI3K/PI3K and p-AKT/AKT, and the increased expression levels of Bax and caspase-3. Overall, this resulted in the inhibition of cell proliferation and promoted cell apoptosis by directly targeting ARRB1. Therefore, miR-379-5p may be a potential target for NSCLC treatment due to its ability to inhibit cell proliferation and accelerate the apoptotic process.

\section{Introduction}

Lung cancer is the leading cause of cancer-related deaths worldwide (1). The 5-year survival rate following diagnosis is estimated to be $\sim 15.6 \%$, which is lower than the rate observed in colon, breast or prostate cancer (2). Non-small cell lung cancer (NSCLC) accounts for $85 \%$ of all lung cancer cases and histologically, it is typically classified in three subtypes: Large-cell neuroendocrine carcinoma, squamous cell carcinoma and adenocarcinoma (3). Over the last several decades, conventional cancer treatments, such as surgery, chemotherapy and radiotherapy, have been used to treat lung cancer (4); however, these therapies do not benefit patients with locally advanced or distant metastatic disease (stage III/IV) (5). Therefore, several molecular targeted therapies have been approved for these patients, including first- and second-generation epidermal growth factor receptor tyrosine kinase inhibitors (6). In total, $>60 \%$ of all newly diagnosed patients with lung cancer have undergone radiotherapy alone and in combination with chemotherapy, targeted therapies and immunotherapy as the first line therapies (7). Thus, there is an urgent requirement to identify promising therapeutic targets that could inhibit cell proliferation and induce cell apoptosis to prevent the progression of lung cancer.

MicroRNAs (miRNAs $/ \mathrm{miR}$ ) are single stranded non-coding RNAs of 19-25 nucleotides in length, that are generated from endogenous hairpin transcripts through a multistep process, which starts in the nucleus and ends in the cytoplasm $(8,9)$. miRNAs are partially complementary to one or more mRNA molecules (10); their main function is to downregulate gene expression in several ways, including translational repression, mRNA cleavage and de-adenylation (11). Accumulating 
scientific evidence has demonstrated that miRNAs serve an important role in cancer progression and treatment. For example, Cazzoli et al (12) screened 742 miRNAs isolated from the circulating exosomes of patients with NSCLC and among them, four miRNAs (miR-378a, miR-379, miR-139-5p and miR-200b-5p) were identified as screening markers to segregate patients with lung adenocarcinoma and granuloma. In addition, miR-21, miR-31 and miR-let7 were discovered to be closely associated with the diagnostic efficacy and survival rate in lung cancer (13). miR-379 is located on human chromosome $14 \mathrm{q} 32$ within a large miRNA gene cluster (14). Previous evidence indicated that miR-379-5p exhibited a tumor-suppressive role in several types of cancer, including osteosarcoma, bladder cancer and melanoma $(15,16)$. Hao et al (17) also reported that miR-379-5p expression levels were significantly downregulated in chemoresistant NSCLC tissues and cells, whereas miR-379-5p overexpression suppressed eukaryotic translation initiation factor $4 \gamma 2$ (EIF4G2) expression to enhance cisplatin chemosensitivity. However, the expression profile and the role of miR-379-5p in NSCLC remains to be fully determined.

$\beta$-Arrestins (ARRBs), including ARRB1 and ARRB2, have been identified as scaffold proteins that mediate the desensitization and internalization of $G$ protein-coupled receptors (GPCRs) (18). Furthermore, ARRBs were discovered to serve as signal transducers, with a previous study demonstrating that they had important roles in several physiological processes, such as chemotaxis, the Frank-Starling force, and pathological conditions including myelofibrosis, pulmonary fibrosis and asthma (19). Emerging data has also suggested that the recruitment of ARRBs may represent a major non-G protein-dependent signaling pathway of GPCRs, such as the endothelin-1 receptor (ET-1R) in cancer (20). In addition, a study on glioblastoma multiforme (GBM) revealed that ARRB1 knockdown suppressed GBM cell proliferation, invasion and glycolysis by inhibiting Src signaling (21). Regarding lung cancer, a previous study indicated that nicotine induced the nuclear translocation of ARRB1, which resulted in the increased expression of proliferative and survival genes, thereby promoting the growth and progression of NSCLC (22). Additionally, Shen et al (23) reported that ARRB1 enhanced the chemosensitivity of lung cancer through the mediation of DNA damage.

The present study aimed to investigate the expression levels of miR-379-5p and ARRB1 in NSCLC. The results indicated that miR-379-5p overexpression may inhibit cell proliferation and promote cell apoptosis by targeting ARRB1. Thereby, this study provided novel insight into lung cancer treatment.

\section{Materials and methods}

Patient studies. The present study was approved by the Ethics Committee of Weinan Central Hospital and written informed consent was obtained from each participant prior to the study initiation. Tumor and para-carcinoma tissues were obtained from 30 patients ( 20 male and 10 female; 13 aged $<50$ and 17 aged $\geq 50$ ) with NSCLC who underwent surgery at Weinan Central Hospital between January 2017 and March 2018. The patients did not undergo any treatment such as chemotherapy or radiotherapy before surgery. The tumor and para-carcinoma tissues were fixed in $10 \%$ formalin at room temperature until use. The clinicopathological features of these patients are presented in Table I.

Plasmids and cell culture. The BEAS-2B, A549, PG49 and DMS-114 cell lines were purchased from the American Type Culture Collection. The cells were cultured in RPMI-1640 medium (Thermo Fisher Scientific, Inc.), supplemented with $10 \%$ FBS (Thermo Fisher Scientific, Inc.), and maintained at $37^{\circ} \mathrm{C}$ in a humidified incubator containing $5 \% \mathrm{CO}_{2}$ until $90 \%$ confluence was reached. The miR-379-5p mimic (5'-UGGUAGACUAUGGAACGUAGG-3'), miR-negative control (NC) mimic (5'-GUGGAUUUUCCUCUA UGAUUU-3'), ARRB1 overexpression pcDNA3.1 plasmid (pcDNA3.1-ARRB1), pcDNA3.1 plasmid (negative control for pcDNA3.1-ARRB1), wild-type (WT) and mutant (MUT) ARRB1 3' untranslated region (UTR) pGL3 plasmids were obtained from Addgene, Inc.

Cell transfection. A549 cells (at $1 \times 10^{4}$ cells/well) were seeded into 96-well plates and subsequently transfected with miR-NC mimics, miR-379-5p mimics, pcDNA3.1 and pcDNA3.1-ARRB1. Untransfected cells were used as the control group. The cells were incubated at $37^{\circ} \mathrm{C}$ and when they reached $70-80 \%$ confluence, they were transfected with 500 ng transfectants using $2.5 \mu 1$ Lipofectamine ${ }^{\circledR} 2000$ reagent (Invitrogen; Thermo Fisher Scientific, Inc.) according to the manufacturer's protocol. Following incubation at $37^{\circ} \mathrm{C}$ for $6 \mathrm{~h}$, the serum-free RPMI-1640 medium was replaced with fresh RPMI-1640 medium containing 10\% FBS, and the cells continued to incubate for $24 \mathrm{~h}$. All experiments were performed in triplicate.

Reverse transcription-quantitative PCR (RT-qPCR). The relative miR-379-5p and $\mathrm{mRNA}$ expression levels were determined using RT-qPCR. Total RNA from tissues and cells was extracted using the RNeasy Mini kit (Qiagen, Inc.), according to the manufacturer's protocol, and the concentration was determined on a Nanodrop 2000 spectrophotometer (Thermo Fisher Scientific, Inc.). Total RNA was reverse-transcribed into cDNA using M-MLV Reverse Transcriptase (Promega Corporation). M-MLV RT 5X reaction buffer (containing Tris- $\mathrm{HCl}, \mathrm{KCl}, \mathrm{MgCl}_{2}$ and DDT; $5 \mu \mathrm{l}$ ) and $5 \mu \mathrm{ldNTP}$ and Oligo DT primer were used for RT. The following temperature protocol was used for the reverse transcription: $43^{\circ} \mathrm{C}$ for $30 \mathrm{~min}, 97^{\circ} \mathrm{C}$ for $5 \mathrm{~min}$ and $5^{\circ} \mathrm{C}$ for $5 \mathrm{~min}$. qPCR was subsequently performed using a PrimeScript ${ }^{\mathrm{TM}}$ RT-PCR kit (Qiagen Inc.) following the manufacturer's protocol. The following thermocycling conditions were used for the qPCR: Initial denaturation at $95^{\circ} \mathrm{C}$ for $6 \mathrm{~min}$; followed by 40 cycles of initiation at $94^{\circ} \mathrm{C}$ for $30 \mathrm{sec}$, annellation at $60^{\circ} \mathrm{C}$ for $30 \mathrm{sec}$ and elongation at $73^{\circ} \mathrm{C}$ for $90 \mathrm{sec}$. The following primer pairs were used for the qPCR: miR-379-5p forward, 5'-GCGCTG GTAGACTATGGAA-3' and reverse, 5'-GTGCAGGGTCCG AGGT-3'; U6 forward, 5'-CTCGCTTCGGCAGCACATATA CT-3' and reverse, 5'-ACGCTTCACGAATTTGCGTGTC-3'; ARRB1 forward, 5'-CCTGGATGTCTTGGGTCTG-3' and reverse, 5'-TGATGGGTTCTCCGTGGTA-3'; Bcl-2 forward, 5'-CTGCACCTGACGCCCTTCACC-3' and reverse, 5'-CAC ATGACCvCCACCGAACTCAAAGA-3'; Bax forward, 
Table I. Association between clinicopathological features of patients with non-small cell lung cancer and miR-379-5p expression levels.

miR-379-5p expression levels

\begin{tabular}{|c|c|c|c|c|}
\hline \multirow[b]{2}{*}{ Variable } & \multirow[b]{2}{*}{$\mathrm{n}$} & & \multirow[b]{2}{*}{ P-value } \\
\hline & & High & Low & \\
\hline Age (years) & & & & 0.8804 \\
\hline$\geq 50$ & 17 & 7 & 10 & \\
\hline$<50$ & 13 & 5 & 8 & \\
\hline Sex & & & & 0.6023 \\
\hline Male & 20 & 8 & 12 & \\
\hline Female & 10 & 5 & 5 & \\
\hline Tumor size & & & & $0.0281^{\mathrm{a}}$ \\
\hline$<3 \mathrm{~cm}$ & 14 & 10 & 4 & \\
\hline$\geq 3 \mathrm{~cm}$ & 16 & 5 & 11 & \\
\hline TNM stage & & & & 0.0510 \\
\hline I-II & 12 & 6 & 6 & \\
\hline III-IV & 18 & 3 & 15 & \\
\hline Metastasis & & & & $0.0371^{\mathrm{a}}$ \\
\hline Negative & 13 & 7 & 6 & \\
\hline Positive & 17 & 3 & 14 & \\
\hline
\end{tabular}

${ }^{\mathrm{a}} \mathrm{P}<0.05$. miR, microRNA.

5'-GACCAGCATGACAGATTTCTACCA-3' and reverse, 5'-AACTGAGACTAAGGCAGA AGATG-3'; caspase-3 forward, 5'-CTCGGTCTGGTACAGATGTCGATG-3' and reverse, 5'-GGTTAACCCGGGTAAGAATGTGCA-3'; and GAPDH forward, 5'-ACACCCACTCCTCCACCTTTG-3' and reverse, 5'-TCCACCACCCTGTTGCTGTAG-3'. All experiments were performed in triplicate. The mRNA expression levels were quantified using the $2^{-\Delta \Delta \mathrm{Cq}}$ method (24). The endogenous expression levels of U6 and GAPDH were used to normalize the miR-379-5p and mRNA expression levels, respectively.

Western blotting. The protein expression levels in tissues and cells were determined using western blotting. Total and phosphorylated protein was extracted from the cells using RIPA lysis buffer (Boster Biological Technology) according to the manufacturer's protocols. Protein was quantified using a bicinchoninic acid protein assay kit (Abbkine Scientific Co., Ltd.) and $20 \mu \mathrm{g}$ protein/lane was separated via 15\% SDS-PAGE. The separated proteins were subsequently transferred onto PVDF membranes (EMD Millipore) and blocked with 5\% skimmed milk for $2 \mathrm{~h}$ at room temperature. The membranes were incubated with the following primary antibodies for $1 \mathrm{~h}$ at room temperature: Anti-ARRB1 (1:1,000; cat. no. ab32099), anti-PI3K (1:1,000; cat. no. ab191606), anti-phosphorylated (p)-PI3K (1:1,1000; cat. no. ab182651), anti-AKT (1:500; cat. no. ab8805), anti-p-AKT (1:1,000; cat. no. ab38449), anti-Bcl-2 (1:1,000; cat. no. ab59348), anti-Bax (1:1,000; cat. no. ab53154), anti-caspase-3 (1:500; cat. no. ab13847) and anti-GAPDH (1:2,500; cat. no. ab9485; all from Abcam). Following the primary antibody incubation, the membranes were incubated with a horseradish peroxidase-conjugated anti-rabbit secondary antibody (1:5,000; cat. no. ab205718; Abcam) for $45 \mathrm{~min}$ at room temperature. The protein bands were visualized using an ECL Western Blotting kit (Abcam) and the expression levels were analyzed using ImageJ software (v1.48U; National Institutes for Health). All experiments were performed in triplicate. GAPDH protein expression levels were used as an internal control.

Cell Counting Kit-8 (CCK-8) assay. The proliferation of transfected A549 cells was assessed using a CCK-8 kit (APeXBIO Technology LLC) according to the manufacturer's protocols. Briefly, the cells at the density of $1 \times 10^{4}$ cells/well were seeded into 96-well plates and following $0,12,24$ or $48 \mathrm{~h}, 10 \mu \mathrm{l} \mathrm{CCK}-8$ reagent was added to each well and incubated for an additional $24 \mathrm{~h}$ in RPMI-1640 medium supplemented with $10 \%$ FBS at $37^{\circ} \mathrm{C}$ in a humidified incubator containing $5 \% \mathrm{CO}_{2}$. Finally, the optical density of each well was measured using a microplate reader at $450 \mathrm{~nm}$.

Flow cytometric analysis of apoptosis. Following transfection, the apoptotic rate of A549 cells was analyzed using flow cytometry. Briefly, $2 \times 10^{5}$ transfected cells were plated into a 24-well plate and incubated for $48 \mathrm{~h}$ at $37^{\circ} \mathrm{C}$ with $5 \% \mathrm{CO}_{2}$. Subsequently, cells were incubated with $10 \mu \mathrm{g} / \mathrm{ml}$ propidium iodide (Sigma-Aldrich; Merck KGaA) and $50 \mu \mathrm{g} / \mathrm{ml}$ Annexin V-FITC (BD Biosciences) at room temperature for $15 \mathrm{~min}$ in the dark. Finally, the stained cells were analyzed using a FACScan flow cytometer (BD Biosciences) carrying CellQuest Pro software (version 6.0; BD Biosciences). The apoptotic cell rate was calculated by the number of (apoptotic cells + death cells)/all cells. 

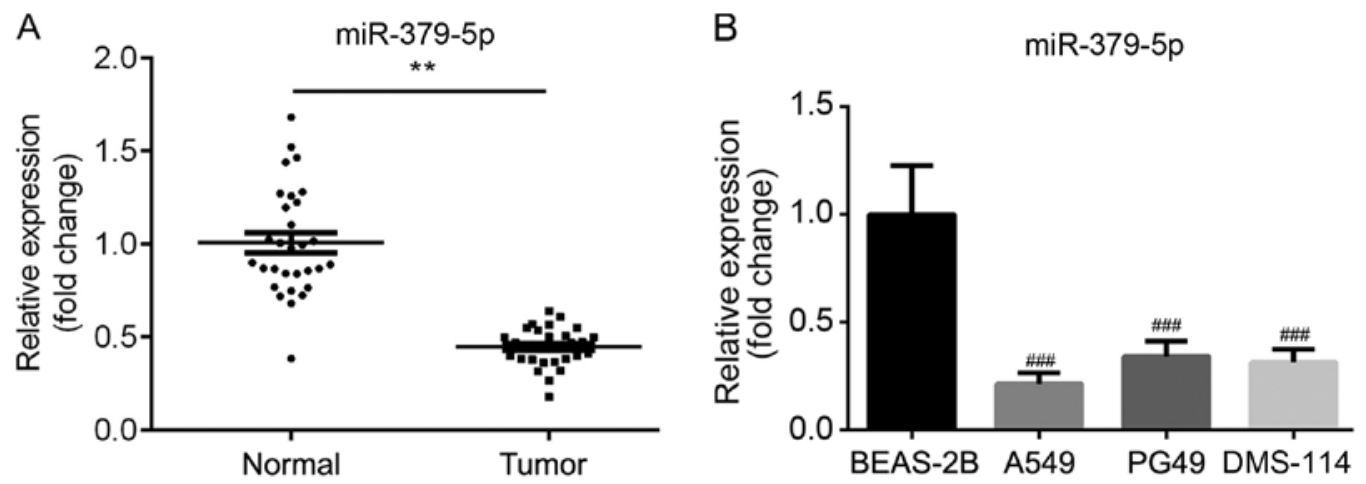

Figure 1. miR-379-5p expression levels are downregulated in NSCLC tissues and cell lines. Expression levels of miR-379-5p in (A) tissues from patients with NSCLC and (B) human bronchial epithelial cell line (BEAS-2B) and NSCLC cell lines (A549, PG49 and DMS-114) were determined using reverse transcription-quantitative PCR. ${ }^{* *} \mathrm{P}<0.01$ vs. normal group; ${ }^{\# \# t} \mathrm{P}<0.001$ vs. BEAS-2B group. miR, microRNA; NSCLC, non-small cell lung cancer.

Bioinformatics analysis and dual-luciferase reporter assay. To predict the target genes of miR-379-5p, bioinformatic online tool TargetScan (http://www.targetscan.org) was employed. After transfection for $24 \mathrm{~h}$, a dual-luciferase reporter assay was performed to verify the direct interaction between ARRB1 and miR-379-5p. A549 cells at the density of $2 \times 10^{4}$ cells/well were seeded into 96 -well plates and incubated for $24 \mathrm{~h}$ at $37^{\circ} \mathrm{C}$ in a humidified atmosphere with $5 \% \mathrm{CO}_{2}$. The cells were subsequently co-transfected with $2.5 \mu \mathrm{g}$ ARRB1-3'UTR-WT or ARRB1-3'UTR-MUT pGL3 plasmids, and miR-379-5p mimic or miR-NC mimic, using $2.5 \mu 1$ Lipofectamine ${ }^{\circledR} 2000$ reagent (Invitrogen; Thermo Fisher Scientific, Inc.), and incubated for $24 \mathrm{~h}$ following the manufacturer's protocol. The luciferase activity of the transfected A549 cells was analyzed using a Dual-Luciferase Reporter Assay kit (BioVision, Inc.), according to the manufacturer's protocol. The firefly luciferase activity was normalized to Renilla luciferase activity.

Statistical analysis. Statistical analysis was performed using GraphPad Prism version 5.01 software (GraphPad Software, Inc.) and all data are presented as the mean \pm SD from three replicates. Statistical differences between the cancer and normal tissues were performed using a paired Student's t-test, whereas the association between the miR-379-5p expression levels and the clinicopathological features were determined using a $\chi^{2}$-test. A one-way ANOVA, followed by Tukey's post hoc test for multiple comparisons was performed to analyze the significant differences among multiple groups. $\mathrm{P}<0.05$ was considered to indicate a statistically significant difference.

\section{Results}

miR-379-5p expression levels are downregulated and ARRBI expression levels are upregulated in NSCLC tissues and cell lines. The expression levels of miR-379-5p in normal and tumor tissues were determined using RT-qPCR. The results revealed that miR-379-5p expression levels were significantly downregulated in the tumor tissues compared with the normal tissues (Fig. 1A). Similarly, the expression levels of miR-379-5p were significantly decreased in NSCLC cells, including A549, PG49 and DMS-114 compared with normal human bronchial epithelial cell line (BEAS-2B; Fig. 1B). The high or low expression levels of miR-379-5p were also discovered to be significantly associated with the tumor size and metastasis, but not with the age, sex or TNM stage (Table I). In addition, the mRNA and protein expression levels of ARRB1 in the tissues and cell lines were determined using RT-qPCR and western blotting, respectively; both the mRNA (Fig. 2A and D) and protein (Fig. 2B, C, E and F) expression levels of ARRB1 were significantly upregulated in tumor tissues and cell lines compared with normal tissues and the BEAS-2B cell line, respectively.

Successful transfection of A549 cells with miR-379-5p mimics. A549 cells with lower miR-379-5p and higher ARRB1 levels compared with other cells were used to transfected with miR-379-5p mimics or miR-NC mimics; compared with the miR-NC mimics group, the expression level of miR-379-5p was significantly upregulated in the miR-379-5p mimics group (Fig. 3). Therefore, the miR-379-5p mimic and miR-NC mimic were used for further experimental procedures.

Successful construction of ARRBI overexpression plasmids. The ARRB1 overexpression plasmids were transfected into A549 cells in order to verify the successful construction of overexpression plasmids. Thus, the mRNA and protein expression levels of ARRB1 were analyzed using RT-qPCR and western blot analysis, respectively. The results demonstrated that the mRNA expression level of ARRB1 was significantly upregulated in the pcDNA3.1-ARRB1 group compared with the pcDNA3.1 group (Fig. 4A). Consistent with the results of ARRB1 mRNA expression levels, western blotting also revealed that the protein expression level of ARRB1 was significantly increased in the pcDNA3.1-ARRB1 group compared with the pcDNA3.1 group (Fig. 4B and C). Thus, the ARRB1 overexpression plasmids were used for further cell experiments.

miR-379-5p overexpression inhibits A549 cell proliferation, while ARRB1 overexpression rescues this inhibition. Following transfection, the proliferation rate of A549 cells was detected at $0,12,24$ and $48 \mathrm{~h}$ using a CCK-8 assay. The proliferation rate was demonstrated to be significantly inhibited in the miR-379-5p mimics group compared with the miR-NC mimics group (Fig. 5). Notably, a significantly increased proliferation rate was observed in the miR-379-5p mimics 

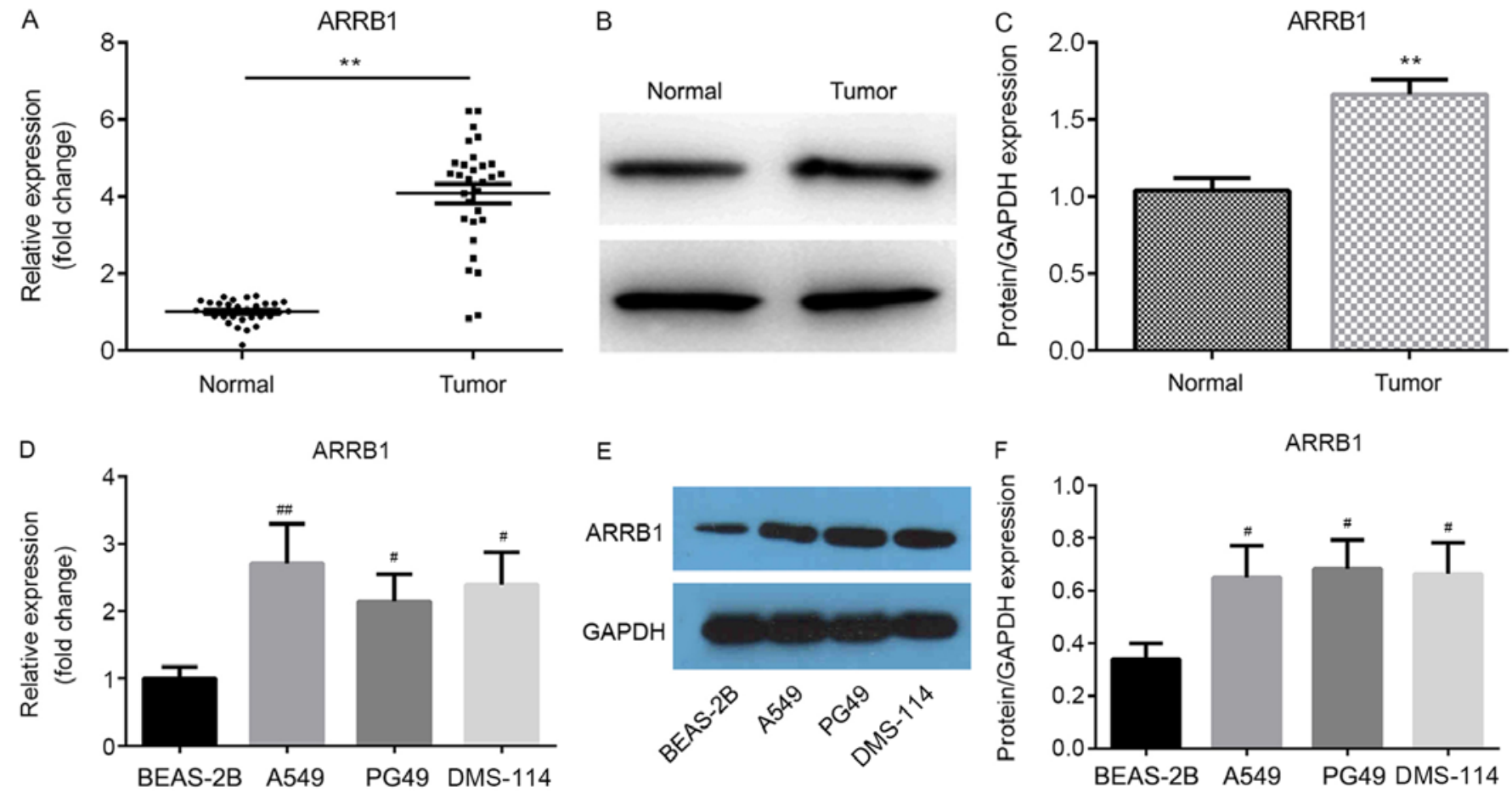

Figure 2. ARRB1 expression levels are upregulated in NSCLC tissues and cells. Expression levels of ARRB1 in tissues from patients with NSCLC were analyzed using (A) RT-qPCR or (B) western blotting. (C) Semi-quantification of ARRB1 expression levels from part B. Expression levels of ARRB1 in NSCLC cell lines were analyzed using (D) RT-qPCR and (E) western blotting. (F) Semi-quantification of ABBR1 expression levels from part E. ${ }^{* *} \mathrm{P}<0.01 \mathrm{vs.}$. he normal group or BEAS-2B group; ${ }^{\#} \mathrm{P}<0.05$ vs. the BEAS-2B group; ${ }^{\# \#} \mathrm{P}<0.01$ vs. the BEAS-2B group.ARRB1, $\beta$-arrestin-1; NSCLC, non-small cell lung cancer; RT-qPCR, reverse transcription-quantitative PCR.

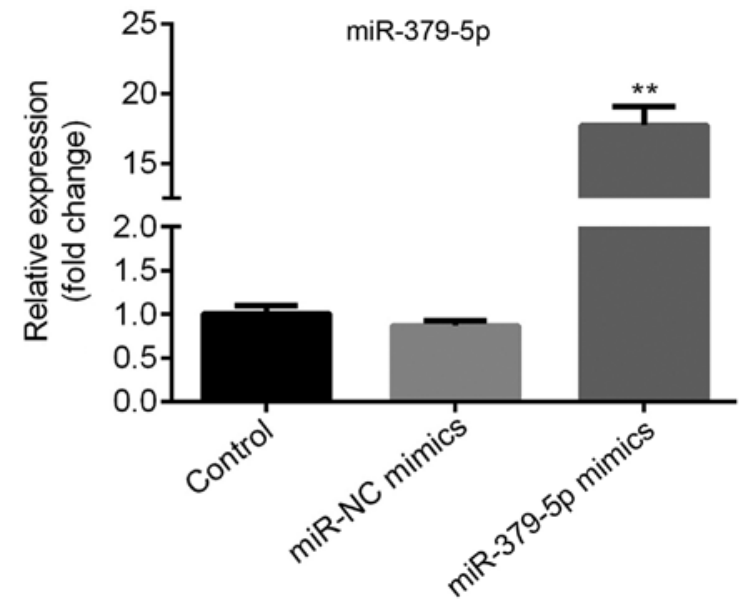

Figure 3. Successful transfection of miR-379-5p mimics and miR-NC mimics. A549 cells were transfected with miR-379-5p mimics or miR-NC mimics. The expression levels of miR-379-5p were determined using reverse transcription-quantitative $\mathrm{PCR}$.* $^{*}<0.01$ vs. the miR-NC mimics group. miR, microRNA; NC, negative control.

+ pcDNA3.1-ARRB1 group compared with the miR-379-5p mimics group (Fig. 5). These results indicated that miR-379-5p overexpression may decrease, while ARRB1 overexpression may increase, the cell proliferation rate.

miR-379-5p overexpression promotes A549 cell apoptosis, while ARRBI overexpression reverses the promotion. Following the transfection with the miR-379-5p mimics, miR-NC mimics or ARRB1 overexpression plasmids, the cells were incubated for $48 \mathrm{~h}$ and the apoptotic cell ratio was determined using flow cytometric analysis. Among the four groups, overexpression of miR-379-5p increased the apoptotic rate, while ARRB1 overexpression decreased the improvement induced by miR-379-5p (Fig. 6A and B). Thus, these findings indicated that miR-379-5p overexpression may induce A549 cell apoptosis, whereas ARRB1 overexpression may reduce cell apoptosis.

miR-379-5p regulates ARRB1, Bcl-2, Bax and caspase-3 $m R N A$ expression levels in NSCLC cells by regulating the expression level of $A R R B 1$. The results of RT-qPCR revealed that ARRB1 and Bcl-2 mRNA expression levels were significantly decreased in the miR-379-5p mimics group compared with the miR-NC mimics group, whereas the expression levels were significantly increased in the miR-379-5p mimics + pcDNA3.1-ARRB1 group compared with the miR-379 mimics group (Fig. 7A and B). In addition, the mRNA expression levels of Bax and caspase-3 were significantly upregulated in the miR-379-5p mimics group; there was no significant difference among the control, miR-NC mimics and miR-379-5p mimics + pcDNA3.1-ARRB1 groups (Fig. 7C and D). These results indicated that miR-379-5p overexpression may downregulate ARRB1 and Bcl-2 expression levels, and upregulate Bax and caspase-3 expression levels, whereas ARRB1 overexpression reversed the downregulation of $\mathrm{Bcl}-2$ and upregulation of $\mathrm{Bax}$ and caspase- 3 .

miR-379-5p regulates the protein expression levels of $A R R B 1$, $P I 3 K / p-P I 3 K, A K T / p-A K T, B c l-2, B a x$ and caspase-3 by regulating the expression level of $A R R B 1$. ARRB1, p-PI3K/ 

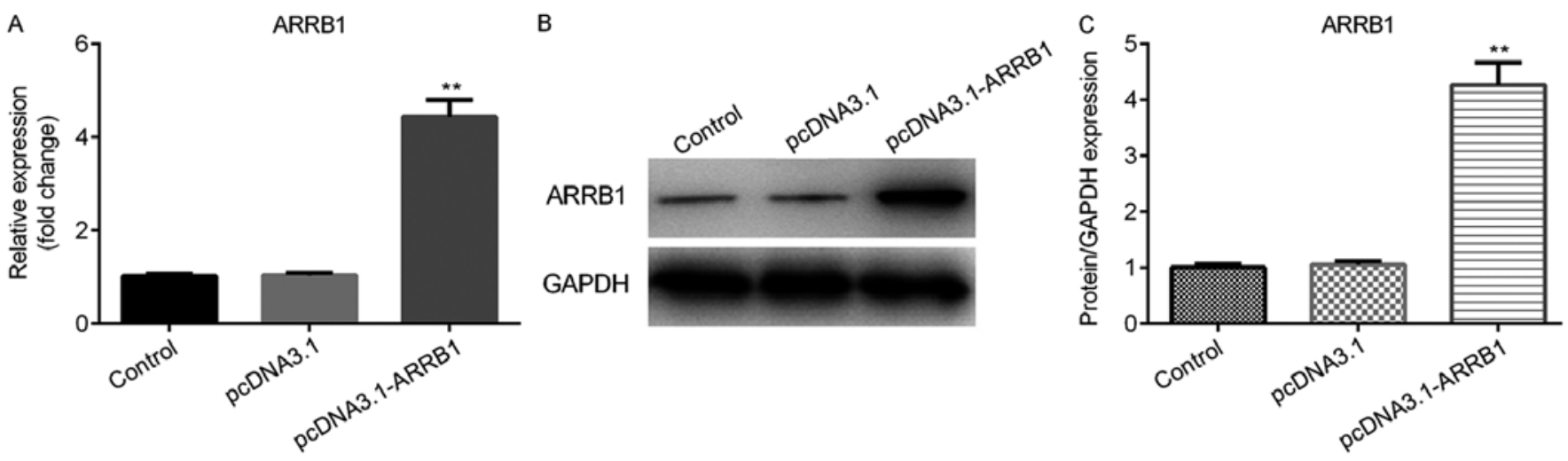

Figure 4. Successful construction of ARRB1 overexpression plasmids. A549 cells were transfected with pcDNA3.1-ARRB1 or pcDNA3.1. The expression levels of ARRB1 were determined using (A) reverse transcription-quantitative PCR and (B) western blotting. (C) Semi-quantification of ARRB1 expression levels from part B. ${ }^{* *} \mathrm{P}<0.01$ vs. the pcDNA3.1 group. ARRB1, $\beta$-arrestin-1.

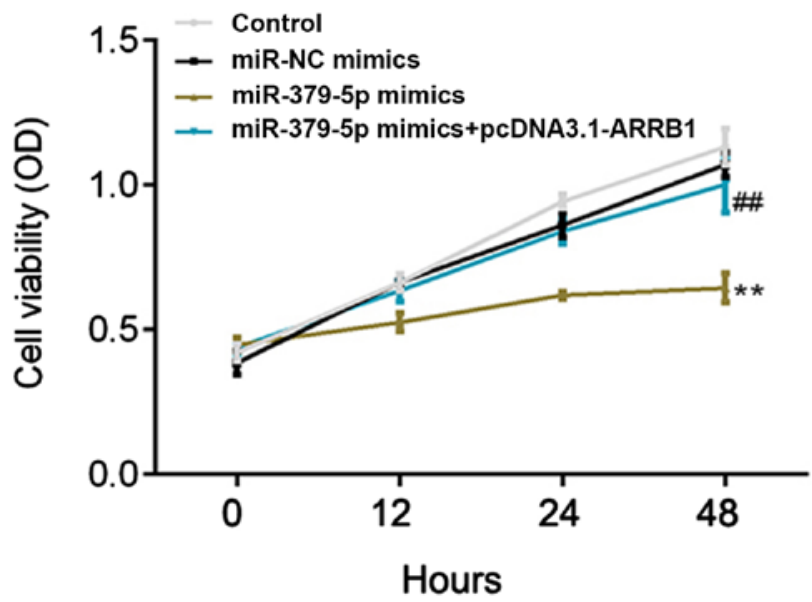

Figure 5. Overexpression of miR-379-5p inhibits the proliferation of A549 cells. The cell proliferation rate of A549 cells following the transfection of miR-NC mimics, miR-379-5p mimics and miR-379-5p mimics + pcDNA3.1-ARRB1 was determined using a Cell Counting Kit- 8 assay. ${ }^{* *} \mathrm{P}<0.01$ vs. the miR-NC mimics group; ${ }^{\# *} \mathrm{P}<0.01$ vs. the miR-379-5p mimics group. miR, microRNA; NC, negative control. miR, microRNA; NC, negative control; ARRB1, $\beta$-arrestin-1.

PI3K, p-AKT/AKT and Bcl-2 expression levels were significantly decreased in the miR-379-5p mimics group compared with the miR-NC mimics group; however, the expression levels of these proteins in the miR-379-5p mimics group were significantly reversed following the overexpression of ARRB1 (Fig. 8A and B). Increased expression levels of Bax and caspase-3 were detected in the miR-379-5p mimics group; however, these expression levels were reversed following the overexpression of ARRB1 (Fig. 8A and B). Therefore, these findings indicated that miR-379-5p may inhibit ARRB1, PI3K/p-PI3K, AKT/p-AKT and Bcl-2 expression levels, while increasing Bax and caspase-3 protein expression levels. In addition, it was determined that the overexpression of ARRB1 may reverse these effects.

miR-379-5p directly targets ARRB1. A dual-luciferase reporter assay was performed to determine whether ARRB1 was a direct target gene of miR-379-5p. The results of the bioinformatics analysis using TargetScan (http://www.targetscan. org) revealed that ARBB1 was a potential direct target gene of miR-379-5p by binding to the ARRB1-3'UTR (Fig. 9A). Subsequently, the dual-luciferase reporter assay demonstrated that the relative luciferase activity was significantly reduced in the ARBB1-3'UTR-WT and miR-379-5p mimics group compared with the miR-NC mimic and ARBB1-3'UTR-WT group (Fig. 9B). Thus, it was verified that miR-379-5p directly targeted ARRB1.

\section{Discussion}

The present study demonstrated that miR-379-5p expression levels were downregulated in NSCLC and that the overexpression of miR-379-5p subsequently reduced the expression levels of ARRB1. Furthermore, miR-379-5p overexpression decreased the expression levels of p-PI3K/PI3K, p-AKT/ $\mathrm{AKT}$ and $\mathrm{Bcl}-2$, and increased the expression levels of Bax and caspase-3 in A549 cells. These findings resulted in the inhibition of cell proliferation and induced cell apoptosis. In addition, a dual-luciferase reporter assay was performed to further verify that miR-379-5p directly targeted ARRB1. The results of the present study revealed that the effects of miR-379-5p on cell proliferation and apoptosis were regulated through the PI3K/AKT signaling pathway by targeting ARRB1. Therefore, these findings indicated that miR-379-5p may suppress NSCLC development by directly inhibiting ARRB1 expression levels, suggesting that it should be further investigated for its potential application in clinical trials.

miR-379-5p has been identified as a tumor suppressor in several types of cancer, including bladder cancer, osteosarcoma and nasopharyngeal carcinoma $(15-16,25)$. In the present study, it was discovered that the expression levels of miR-379-5p were downregulated in NSCLC, which was consistent with a previous study by Hao et al (17). In addition, consistent with the findings of Dasgupta et al (22), ARRB1 expression levels were also revealed to be upregulated in NSCLC. ARRBs, multifunctional adapter proteins, are most commonly known as regulators of GPCR signaling, and they are often found upregulated in multiple types of human cancer $(26,27)$. Furthermore, several signaling pathways have been reported to be regulated by ARRB1, including AMPK, HIF1A and PI3K/ 

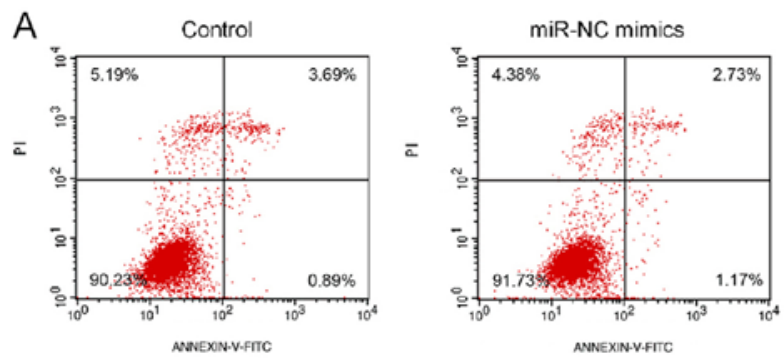

miR-379-5p mimics+
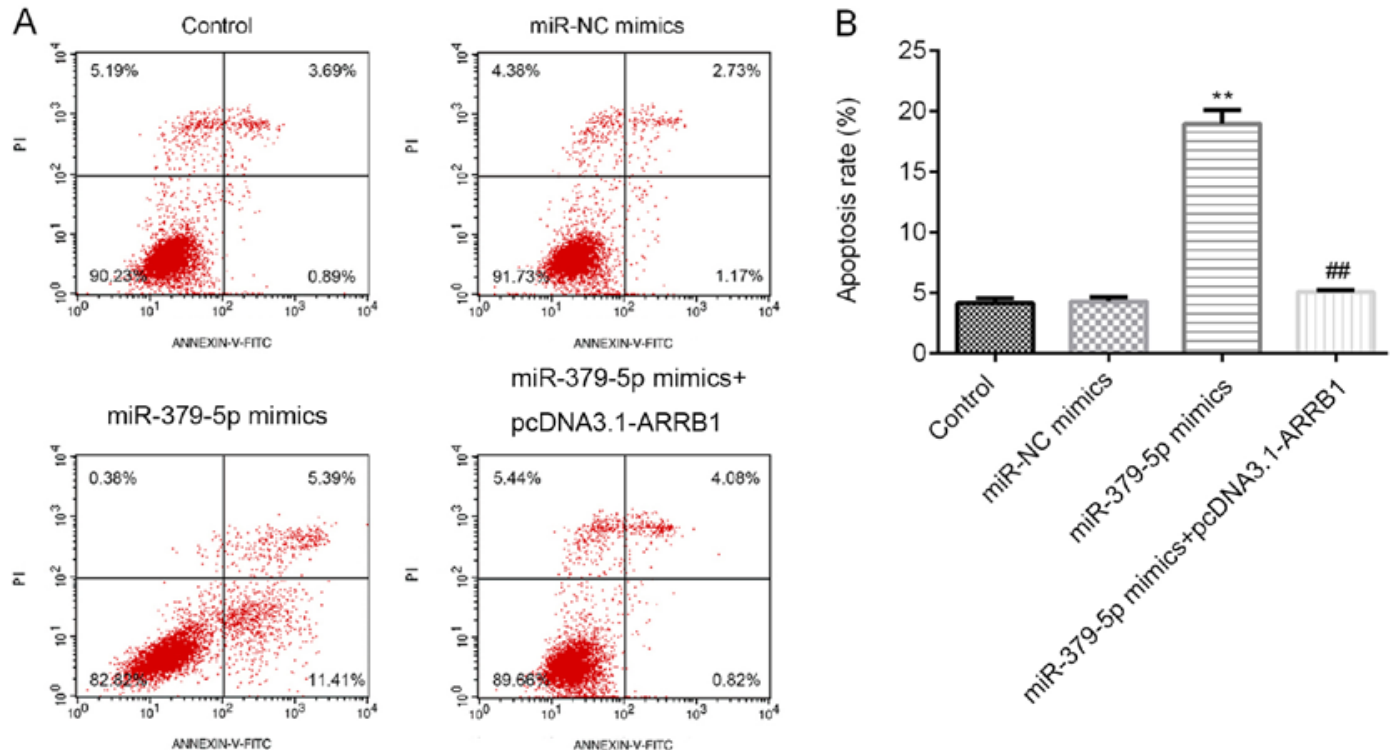

Figure 6. miR-379-5p promotes the apoptosis of A549 cells. (A) Flow cytometric analysis of apoptosis in A549 cells transfected with the miR-NC mimics, miR-379-5p mimics and miR-379-5p mimics + pcDNA3.1-ARRB1. Q1, living cells; Q2, necrotic cells; Q3, late apoptosis; Q4, early apoptosis. (B) Semi-quantitative analysis of cell apoptosis from part A. ${ }^{* *} \mathrm{P}<0.01$ vs. the miR-NC mimics group; ${ }^{\# \#} \mathrm{P}<0.01 \mathrm{vs}$. the miR-379-5p mimics group. miR, microRNA; NC, negative control; ARRB1, $\beta$-arrestin-1; PI, propidium iodide.
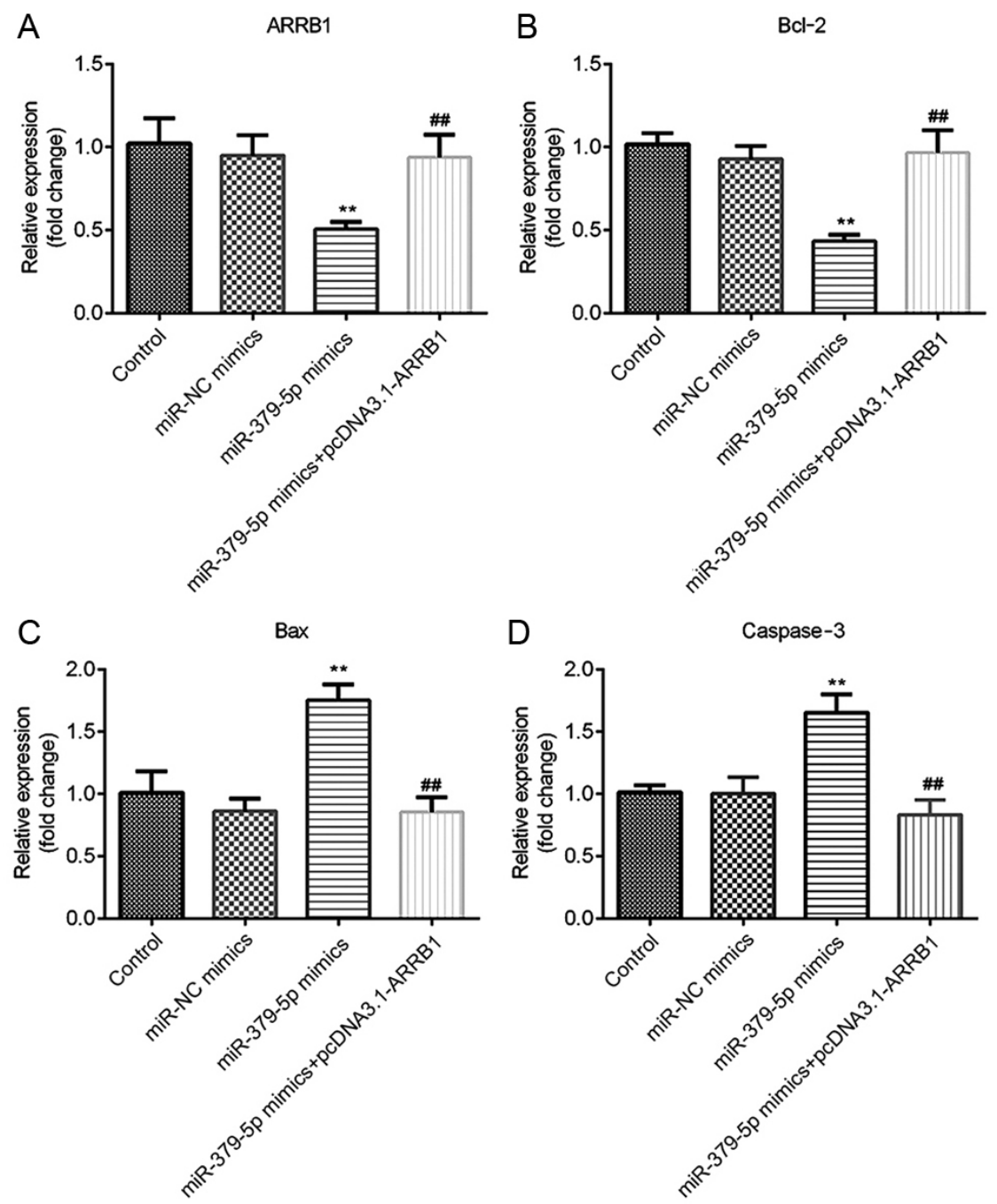

Figure 7. Expression levels of ARRB1 and Bcl-2 are downregulated, whereas the expression levels of Bax and caspase-3 are upregulated in the miR-379-5p mimics group. Expression levels of (A) ARRB1, (B) Bcl-2, (C) Bax and (D) caspase-3 were analyzed using reverse transcription-quantitative PCR. ** $\mathrm{P}<0.01$ vs. the miR-NC mimics group; ${ }^{\# /} \mathrm{P}<0.01$ vs. the miR-379-5p mimics group. miR, microRNA; NC, negative control; ARRB1, $\beta$-arrestin-1. 

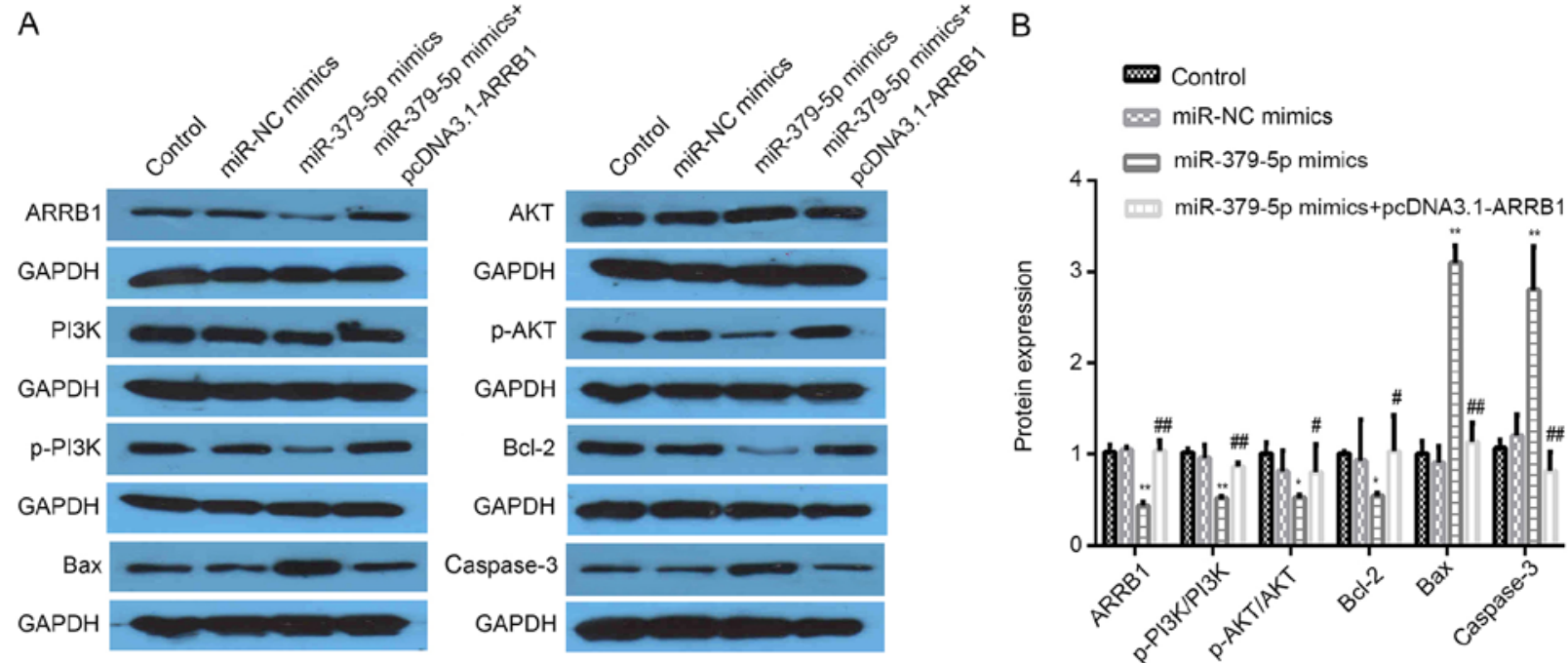

Figure 8. miR-379-5p mimics downregulate the expression levels of ARRB1, p-PI3K/PI3K, p-AKT/AKT and Bcl-2 and upregulate the expression levels of Bax and caspase-3. (A) Expression levels of ARRB1, p-PI3K/PI3K, p-AKT/AKT, Bcl-2, Bax and caspase-3 protein were analyzed using western blotting. Each blot is accompanied by its loading control GAPDH. (B) Semi-quantification of the expression levels from part A. ${ }^{*} \mathrm{P}<0.05$ vs. the miR-NC mimics group; ${ }^{* *} \mathrm{P}<0.01$ vs. the miR-NC mimics group; ${ }^{*} \mathrm{P}<0.05$ vs. the miR-379-5p mimics group; ${ }^{\# \#} \mathrm{P}<0.01$ vs. the miR-379-5p mimics group. miR, microRNA; NC, negative control; ARRB1, $\beta$-arrestin-1; p-, phosphorylated.

\section{A Position 107-113 of ARRB1 3' UTR 5' ...UUUGUUCUUCCAGUUUCUACCAG... hsa-miR-379-5p 3' GgaugcaAgGuaucagauggu Mutant position of ARRB1 3' UTR $\quad 5^{\prime}$...UUUGUUCUUCCAGUUGGACAGG...}

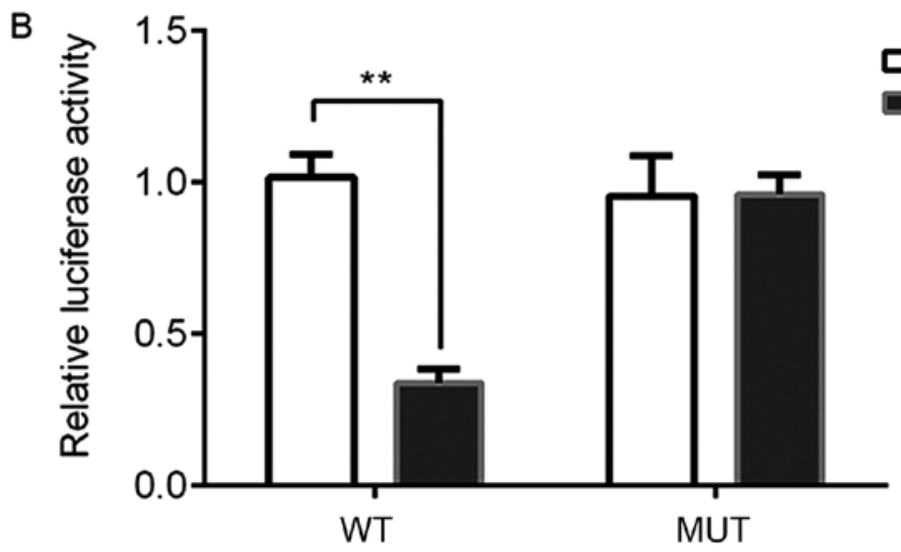

Figure 9. ARRB1 is a target gene of miR-379-5p. (A) Bioinformatical analysis using TargetScan revealed the target prediction binding site of miR-379-5p in the ARRB1 3'UTR. (B) Relative luciferase activity was analyzed using a dual-luciferase reporter assay following the co-transfection of WT or MUT ARRB1 pGL3 plasmid with the miR-NC mimics or miR-379-5p mimics. "** $<0.01$ vs. the miR-NC mimics group. miR, microRNA; NC, negative control; ARRB1, $\beta$-arrestin-1; UTR, untranslated region; MUT, mutant; WT, wild-type.

AKT $(27,28)$. The results of the present study also revealed that ARRB1 downregulation induced by miR-379-5p overexpression inhibited the expression levels of PI3K/AKT and Bcl-2, whereas Bax and caspase-3 expression levels were increased. These results are consistent with those reported by Zhang et al (29). Zhan et al (30), suggested that ARRB1 served a vital role in inhibiting cell apoptosis. This finding is strongly supported by the results of the present study, as the miR-379-5p-induced downregulation of ARRB1 resulted in the inhibition of cell proliferation and increased rates of cell apoptosis.
A previous study by Rosanò and Bagnato (20) revealed that ET-1R is a regulator of cell proliferation, survival, motility, cytoskeletal changes, angiogenesis, metastasis and drug resistance. Additionally, the study revealed that the functions of ET-1R were strongly regulated by ARRB1 (20). In addition, the tumor-related effects of ARRB1 were associated with the capacity of the cells to migrate, invade, proliferate, undergo apoptosis and to manage drug resistance (20). Thus, the effects of ARRB1 in lung cancer should be further investigated. The present study hypothesized that the miR-379-5p targeting of ARRB1 may represent a promising therapeutic target in 
NSCLC, thus, further studies should focus on the effects of miR-379-5p on cell migration, invasion and drug resistance by targeting ARRB1. For example, the targeting of ARRB1 by miR-379-5p may suppress cancer progression and enhance the efficacy of chemotherapy, which may subsequently improve NSCLC prognosis.

However, the present study was limited by the fact that the complete underlying molecular mechanism was not determined. Thus, the involvement of other signaling pathways or related factors influenced by miR-379-5p in regulating the proliferation and apoptosis of lung cancer cells remains to be further investigated, which is the aim of our future studies.

In conclusion, the present study revealed that miR-379-5p and ARRB1 expression levels were downregulated and upregulated in NSCLC, respectively. Furthermore, the overexpression of miR-379-5p was discovered to downregulate the expression levels of ARRB1, p-PI3K/PI3K, p-AKT/ $\mathrm{AKT}$ and $\mathrm{Bcl}-2$, and upregulate the expression levels of Bax and caspase-3, thus resulting in the inhibition of cell proliferation and the promotion of cell apoptosis. Thus, it was hypothesized that miR-379-5p may serve as a tumor suppressor in NSCLC by directly targeting ARRB1. Overall, miR-379-5p may be considered as a potential therapeutic target for patients with NSCLC to prevent the progress of the disease.

\section{Acknowledgements}

Not applicable.

\section{Funding}

No funding was received.

\section{Availability of data and materials}

The datasets used during the present study are available from the corresponding author upon reasonable request.

\section{Authors' contributions}

YJ and AW contributed to study design. YJ, PZ and YG performed all experiments and data analysis. YJ was a major contributor in writing the manuscript. All authors read and approved the final manuscript.

\section{Ethics approval and consent to participate}

The present study was approved by the Ethics Committee of Weinan Central Hospital and written informed consent was obtained from each participant prior to the study initiation.

\section{Patient consent for publication}

Not applicable.

\section{Competing interests}

The authors declare that they have no competing interests.

\section{References}

1. Chen R, Xia W, Wang S, Xu Y, Ma Z, Xu W, Zhang E, Wang J, Fang T, Zhang Q, et al: Long noncoding RNA SBF2-AS1 is critical for tumorigenesis of early-stage lung adenocarcinoma. Mol Ther Nucleic Acids 16: 543-553, 2019.

2. Nanavaty P, Alvarez MS and Alberts WM: Lung cancer screening: Advantages, controversies, and applications. Cancer Control 21: 9-14, 2014.

3. Richtmann S, Wilkens D, Warth A, Lasitschka F, Winter H, Christopoulos P, Herth FJF, Muley T, Meister $M$ and Schneider MA: FAM83A and FAM83B as prognostic biomarkers and potential new therapeutic targets in NSCLC. Cancers (Basel) 11: 652, 2019.

4. Lemjabbar-Alaoui H, Hassan OU, Yang YW and Buchanan P: Lung Cancer: Biology and treatment options. Biochim Biophys Acta 1856: 189-210, 2015.

5. Zhang T, Song X, Liao X, Wang X, Zhu G, Yang C and Xie X: Distinct prognostic values of phospholipase $\mathrm{C}$ beta family members for non-small cell lung carcinoma. Biomed Res Int 2019: 4256524, 2019.

6. Castellanos-Rizaldos E, Zhang X, Tadigotla VR, Grimm DG, Karlovich C, Raez LE and Skog JK: Exosome-based detection of activating and resistance EGFR mutations from plasma of non-small cell lung cancer patients. Oncotarget 10: 2911-2920, 2019.

7. Liu C, Hu Q, Xu B, Hu X, Su H, Li Q, Zhang X, Yue J and Yu J: Peripheral memory and naïve $\mathrm{T}$ cells in non-small cell lung cancer patients with lung metastases undergoing stereotactic body radiotherapy: Predictors of early tumor response. Cancer. Cell Int 19: 121, 2019.

8. Hammond SM: An overview of microRNAs. Adv Drug Deliv Rev 87: 3-14, 2015.

9. Di Leva G, Garofalo M and Croce CM: MicroRNAs in cancer. Annu Rev Pathol 9: 287-314, 2014.

10. Gebert LFR and MacRae IJ: Regulation of microRNA function in animals. Nat Rev Mol Cell Biol 20: 21-37, 2019.

11. Liu B, Li J and Cairns MJ: Identifying miRNAs, targets and functions. Brief Bioinform 15: 1-19, 2014

12. Cazzoli R, Buttitta F, Nicola MD, Malatesta S, Marchetti A, Rom WN and Pass HI: Micrornas derived from circulating exosomes as noninvasive biomarkers for screening and diagnosing lung cancer. J Thorac Oncol 8: 1156-1162, 2013.

13. Wang S, Wang Z, Wang Q, Cui Y and Luo S: Clinical significance of the expression of miRNA-21, miRNA-31 and miRNA-let7 in patients with lung cancer. Saudi J Biol Sci 26: 777-781, 2019.

14. Zhao X and Chu J: MicroRNA-379 suppresses cell proliferation, migration and invasion in nasopharyngeal carcinoma by targeting tumor protein D52. Exp Ther Med 16: 1232-1240, 2018.

15. Wu D, Niu X, Tao J, Li P, Lu Q, Xu A, Chen W and Wang Z: MicroRNA-379-5p plays a tumor-suppressive role in human bladder cancer growth and metastasis by directly targeting MDM2. Oncol Rep 37: 3502-3508, 2017.

16. Xie X, Li YS, Xiao WF, Deng ZH, He HB, Liu Q and Luo W: MicroRNA-379 inhibits the proliferation, migration and invasion of human osteosarcoma cells by targetting EIF4G2. Biosci Rep 37: BSR20160542, 2017.

17. Hao GJ, Hao HJ, Ding YH, Wen H, Li XF, Wang QR and Zhang BB: Suppression of EIF4G2 by miR-379 potentiates the cisplatin chemosensitivity in non-small cell lung cancer cells. FEBS Lett 591: 636-645, 2017.

18. Sun JC, Liu B, Zhang RW, Jiao PL, Tan X, Wang YK and Wang WZ: Overexpression of $\beta$-arrestin1 in the rostral ventrolateral medulla downregulates angiotensin receptor and lowers blood pressure in hypertension. Front Physiol 9: 297, 2018.

19. Kim J, Grotegut CA, Wisler JW, Li T, Mao L, Chen M, Chen W, Rosenberg PB, Rockman HA and Lefkowitz RJ: $\beta$-Arrestin 1 regulates $\beta 2$-adrenergic receptor-mediated skeletal muscle hypertrophy and contractility. Skelet Muscle 8: 39, 2018.

20. Rosanò $L$ and Bagnato $A: \beta$-arrestin1 at the cross-road of endothelin-1 signaling in cancer. J Exp Clin Cancer Res 35: 121, 2016.

21. Lan T, Wang $\mathrm{H}$, Zhang Z, Zhang $\mathrm{M}$, Qu Y, Zhao Z, Fan X, Zhan Q, Song Y and Yu C: Downregulation of $\beta$-arrestin 1 suppresses glioblastoma cell malignant progression via inhibition of src signaling. Exp Cell Res 357: 51-58, 2017.

22. Dasgupta P, Rizwani W, Pillai S, Davis R, Banerjee S, Hug K, Lloyd M, Coppola D, Haura E and Chellappan SP: ARRB1-mediated regulation of E2F target genes in nicotine-induced growth of lung tumors. J Natl Cancer Inst 103: 317-333, 2011. 
23. Shen H, Wang L, Zhang J, Dong W, Zhang T, Ni Y, Cao H, Wang K, Li Y, Wang Y and Du J: ARRB1 enhances the chemosensitivity of lung cancer through the mediation of DNA damage response. Oncol Rep 37: 761-767, 2017.

24. Livak KJ and Schmittgen TD: Analysis of relative gene expression data using real-time quantitative PCR and the 2(-Delta Delta C(T)) method. Methods 25: 402-408, 2001.

25. Zhao X and Chu J: MicroRNA-379 suppresses cell proliferation, migration and invasion in nasopharyngeal carcinoma by targeting tumor protein D52. Exp Ther Med 16: 1232-1240, 2018.

26. Ma Z, Yu YR, Badea CT, Kovacs JJ, Xiong X, Comhair S, Piantadosi CA and Rajagopal S: Vascular endothelial growth factor receptor 3 regulates endothelial function through $\beta$-arrestin. Circulation 139: 1629-1642, 2019.

27. Zecchini V, Madhu B, Russell R, Gomes NP, Warren A, Gaude E, BorlidoJ, Stark R, Zecchini HI, Rao R, et al: Nuclear ARRB1 induces pseudohypoxia and cellular metabolism reprogramming in prostate cancer. EMBO J 33: 1365-1382, 2014.
28. Son D, Kim Y, Lim S, Kang HG, Kim DH, Park JW, Cheong W, Kong HK, Han W, Park WY, et al: MiR-374a-5p promotes tumor progression by targeting ARRB1 in triple negative breast cancer. Cancer Lett 454: 224-233, 2019.

29. Zhang Z, Zhong X, Xiao Y and Chen C: MicroRNA-296 inhibits colorectal cancer cell grow th and enhances apoptosis by targeting ARRB1-mediated AKT activation. Oncol Rep 41: 619-629, 2019.

30. Zhan Y, Xu C, Liu Z, Yang Y, Tan S, Yang Y, Jiang J, Liu H, Chen $\mathrm{J}$ and Wu B: $\beta$-Arrestin1 inhibits chemotherapy-induced intestinal stem cell apoptosis and mucositis. Cell Death Dis 7: e2229, 2016.

(D) $\Theta$ This work is licensed under a Creative Commons Attribution-NonCommercial-NoDerivatives 4.0 International (CC BY-NC-ND 4.0) License. 